\title{
Biphasic survival analysis of trypanotolerance QTL in mice
}

\author{
OD Koudandé ${ }^{1}$, PC Thomson ${ }^{2}$, H Bovenhuis ${ }^{3}$, F Iraqi ${ }^{4,5}$, JP Gibson ${ }^{4,6}$ and JAM van Arendonk ${ }^{3}$ \\ ${ }^{1}$ Institut National des Recherches Agricoles du Bénin, Cotonou, Bénin; ${ }^{2}$ Faculty of Veterinary Science, Centre for Advanced Technologies \\ in Animal Genetics and Reproduction, University of Sydney, Camden, New South Wales, Australia; ${ }^{3}$ Animal Breeding and Genetics \\ Group, Department of Animal Sciences, Wageningen University, AH Wageningen, The Netherlands and ${ }^{4}$ International Livestock \\ Research Institute (ILRI), Nairobi, Kenya
}

\begin{abstract}
A marker-assisted introgression (MAl) experiment was conducted to transfer trypanotolerance quantitative trait loci (QTL) from a donor mouse strain, C57BL/6, into a recipient mouse strain, A/J. The objective was to assess the effect of three previously identified chromosomal regions on mouse chromosomes 1 (MMU1), 5 (MMU5) and 17 (MMU17) in different genetic backgrounds on the survival pattern following infection with Trypanosoma congolense. An exploratory data analysis revealed a biphasic pattern of time to death, with highly distinct early and late mortality phases. In this paper, we present survival analysis methods that account for the biphasic mortality pattern and results of reanalyzing the data from the MAl experiment. The analysis with a Weibull mixture model confirmed the biphasic pattern of time to death. Mortality phase, an unobserved variable, appears to be an important
\end{abstract}

factor influencing survival time and is modeled as a binary outcome variable using logistic regression analysis. Accounting for this biphasic pattern in the analysis reveals that a previously observed sex effect on average survival is rather an effect on proportion of mice in the two mortality phases. The C57BL/6 (donor) QTL alleles on MMU1 and MMU17 act dominantly in the late mortality phase while the A/J (recipient) QTL allele on MMU17 acts dominantly in the early mortality phase. From this study, we found clear evidence for a biphasic survival pattern and provided models for its analysis. These models can also be used when studying defense mechanisms against other pathogens. Finally, these approaches provide further information on the nature of gene actions.

Heredity (2008) 100, 407-414; doi:10.1038/sj.hdy.6801096; published online 6 February 2008

Keywords: mice; trypanotolerance QTL; introgression; trait dissection; QTL effects

\section{Introduction}

Trypanosomosis is a parasitic disease affecting all domesticated animals (cattle, sheep, goats, pigs, dogs, cats, horses, donkeys and camels) as well as humans (sleeping sickness), mice and wild animals. It is a vectorborne disease and tsetse flies are the major vectors in which trypanosomes accomplish part of their life cycle. In domesticated livestock in Africa, different trypanosome species are involved and the disease shows large variation in severity and duration. This variation is associated with both the host species and the trypanosome species. Trypanotolerance is defined as the ability of individuals to withstand trypanosome infection. This ability is an innate feature of the Longhorn N'Dama and other Shorthorn cattle breeds in West Africa (Roberts and Gray, 1973; Roelants, 1986).

Correspondence: Dr OD Koudandé, Institut National des Recherches Agricoles du Bénin, Rue 231, République du Bénin, Cotonou, Littoral 01 BP 884, Benin.

E-mail: dkoud2002@yahoo.fr

${ }^{5}$ Current address: Department of Human Microbiology, Sackler Faculty of Medicine, Tel Aviv University, Ramat Aviv, Tel Aviv 69978, Isarel.

${ }^{6}$ Current address: The Institute for Genetics and Bioinformatics, The University of New England, Armidale, NSW 2351, Australia.

Received 3 July 2007; revised 11 December 2007; accepted 14 December 2007; published online 6 February 2008
A mouse model for trypanotolerance was identified by Morrison et al. (1978), who showed that there was a large variation in resistance to Trypanosoma congolense challenge among laboratory mouse strains. The mouse strain C57BL/ 6 appeared to be the most resistant with mean survival time of 110.2 days whereas the A/J strain, with mean survival of 15.8 days, was highly susceptible. On the basis of an F2 population, Kemp et al. (1996, 1997) identified three chromosomal regions that are associated with trypanotolerance in mice. Although the survival times vary considerably between different mouse strains following challenge, the infection with $T$. congolense results in the eventual death of most mice in all strains (Jennings et al., 1978; Morrison and Murray, 1979; Teale et al., 1999). It appears that the trait generally measured to characterize resistance to trypanosome infection in mice is 'time to death' after infection. Since 1978, most of the resistance studies on trypanosome infection in mice have been using survival measurements as monitors (Kemp et al., 1996, 1997; Clapcott et al., 2000; Iraqi et al., 2000).

Koudandé et al. (2005) reported the successful markerassisted introgression (MAI) of trypanotolerance quantitative trait loci (QTL) alleles from the mouse strain C57BL/6 (donor) into the mouse strain A/J (recipient). They found two phases of mortality when analyzing 'time to death' following trypanosome infection of mice. In the current paper, we present survival analysis 
methods that take into account the biphasic mortality pattern and results of reanalyzing the data set generated by Koudandé et al. (2005). The methods should prove applicable to the investigation of multiphasic processes in biological systems generally.

\section{Materials and methods}

\section{Data}

The data set used in this analysis originates from the MAI experiment reported by Koudandé et al. (2005). This experiment was undertaken to introgress three identified chromosomal regions on mouse chromosome 1 (MMU1), 5 (MMU5) and 17 (MMU17) from the donor C57BL/6 line to the recipient $\mathrm{A} / \mathrm{J}$ line. These lines were chosen because among laboratory mouse lines showing variation in resistance to $T$. congolense, the C57BL/6 line appears the most resistant whereas the $\mathrm{A} / \mathrm{J}$ line shows the lowest level of resistance (Morrison et al., 1978). Chromosomal regions were chosen based on the work of Kemp et al. (1996, 1997).

A reciprocal cross was performed between 20 founder parent A/J and 20 founder parent C57BL/6 to produce $\mathrm{F}_{1}$ mice. This step was followed by four backcross generations $(\mathrm{BC})$ to the recipient line $\mathrm{A} / \mathrm{J}$. For the first backcross generation, all $F_{1}$ mice were crossed with $A / J$ mice. From thereon, candidate individuals for the subsequent backcross steps were selected based on marker information. Three lines of mice, each carrying one of the three C57BL/ 6 donor chromosomal regions, were selected and maintained along the introgression process. At the end of the backcross phase, two generations of inter se mating were performed to fix different QTL alleles within each line. Concurrently, mice that have not shown any marker alleles from the C57BL/ 6 donor line on the three chromosomal regions were selected as internal control. In the last generation, each of the synthetic lines was backcrossed to both parental lines giving a total of 10 groups of mice plus the two parental lines as external controls (Table 1). More details on the MAI scheme are given by Koudandé et al. (2005).
Selection during the backcross and intercross phases was done through genotyping results. Three microsatellite markers were used for each chromosomal region: D1Mit87, D1Mit217 and D1Mit60 for MMU1; D5Mit200, D5Mit113 and D5Mit10 for MMU5; and D17Mit29, D17Mit16 and D17Mit11 for MMU17. The length of the marked regions was $5 \mathrm{cM}$ for chromosome $1,18 \mathrm{cM}$ for chromosome 5 and $7 \mathrm{cM}$ for chromosome 17 (Iraqi et al., 2000). All these microsatellite markers were fixed at alternative alleles for the A/J and C57BL/6 strains.

At the end of the introgression experiment, individuals from the 12 different groups of mice with specific combinations of introgressed chromosomal regions and background C57BL/ 6 donor genotypes were challenged with T. congolense, using the IL1180 clone (Masake et al., 1983). The infecting solution was adjusted at $0.5 \times 10^{5}$ parasites per $\mathrm{ml}$ so that approximately $10^{4}$ parasites were injected to each mouse (intraperitoneal) under a volume of $0.2 \mathrm{ml}$ of solution. The age at which animals were challenged ranged from 9 to 18 weeks. The parasitemia in mice was checked from the fourth through the fourteenth day post-inoculation by means of fresh blood smears observed through a microscope. Two weeks after inoculation, 36 animals showed to be not parasitemic and were discarded from further analyses. The infection rate was $95 \%$ over 731 inoculated mice. The noninfected mice originated from group 9 (eight mice), group 6 (six mice), groups 2 and 5 (four mice for each group), groups 4,8 and $\mathrm{C} 57 \mathrm{BL} / 6$ (three mice for each), groups 3 and $A / J$ (two mice for each) and group 10 (one mouse) and they showed a strong tendency to be associated with crossbred groups. Only data from mice that tested positive for infection during this checking period were used for subsequent analyses. The trait analyzed in this study was 'time to death', that is, the number of days from challenge to the death of each individual mouse. Other variables recorded were age at challenge, sex and batch that is the three distinct periods during which the mice were challenged.

More details on the challenge experiment and their results are described in Koudandé et al. (2005).

Table 1 Genotypes at the three chromosomal regions on MMU1, MMU5 and MMU17, the number of infected mice, proportion of C56BL/6 background genotype, the number of dead mice within 20 days post-infection and description of different groups of mice $(\mathrm{q}=\mathrm{A} / \mathrm{J}$ recipient allele, $\mathrm{Q}=\mathrm{C} 57 \mathrm{BL} / 6$ donor allele)

\begin{tabular}{lcccccl}
\hline Group & \multicolumn{3}{c}{ Genotype } & $\begin{array}{c}\text { Donor C57BL/6 background } \\
\text { genotype }\end{array}$ & $\begin{array}{c}\text { Number of } \\
\text { infected mice }\end{array}$ & Description \\
\cline { 2 - 4 } & MMU1 & MMU5 & MMU17 & & & \\
\hline A/J & qq & qq & qq & 0.000 & 78 & Control recipient line A/J from stock \\
1 & QQ & qq & qq & 0.031 & 60 & Homozygous on MMU1 for donor alleles \\
2 & qQ & qq & qq & 0.015 & 80 & Cross of group 1 with the recipient A/J \\
3 & QQ & qQ & qQ & 0.515 & 52 & Cross of group 1 with the donor C57BL/6 \\
4 & qq & QQ & qq & 0.031 & 63 & Homozygous on MMU5 for donor alleles \\
5 & qq & qQ & qq & 0.015 & 70 & Cross of group 4 with the recipient A/J \\
6 & qQ & QQ & qQ & 0.515 & 56 & Cross of group 4 with the donor C57BL/6 \\
7 & qq & qq & QQ & 0.031 & 21 & Homozygous on MMU17 for donor alleles \\
8 & qq & Qq & qQ & 0.015 & 51 & Cross of group 7 with the recipient A/J \\
9 & qQ & qQ & QQ & 0.515 & 29 & Cross of group 7 with the donor C57BL/6 \\
10 & qq & qq & qq & 0.031 & 58 & Internal control \\
C57BL $/ 6$ & QQ & QQ & QQ & 1.000 & 77 & Control donor line C57BL/6 from stock \\
\hline
\end{tabular}

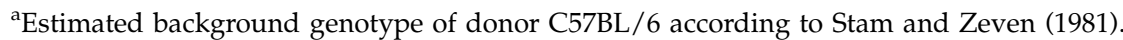

Source: this table is a combination of Tables 2 and 3 from Koudandé et al. (2005), where group 0 and group 11 correspond here respectively to group A/J and group C57BL/6 in this paper, all other groups are identical. 
Statistical analysis

Initial exploratory analyses including Kaplan-Meier survival curves indicated two distinct mortality phases: early (shortly after challenge) and late. To accommodate this, a model comprising a mixture of two survival time distributions was fitted to the data, with the components of the mixture corresponding to the two phases. Although the nonparametric Cox proportional hazards model is the more commonly adopted method of survival analysis, fitting a mixture of survival models requires specification of a fully parametric model, and for this, the Weibull distribution was used. This distribution was chosen on the basis of its simple form, and adequate fit to the survival time data. Fitting of these survival models allows differences in survival across genetic groups to be explored, adjusting for other potential covariates (Kalbfleisch and Prentice, 1980).

\section{Weibull distribution}

The survivor function is given by the expression $S(t)=P(T>t)=\exp \left[-(t / \kappa)^{\rho}\right]$ and the density function is $f(t)=(\rho / \kappa)(t / \kappa)^{\rho-1} \exp \left[-(t / \kappa)^{\rho}\right]$, where $\rho$ is the shape parameter, and $\kappa$ the scale parameter. Since $\log [-\log S(t)]=-\rho \log \kappa+\rho \log t$, a plot of the empirical $\log [-\log \hat{S}(t)]$ versus $\log t$ should be linear, if the Weibull model is appropriate, with the slope being the shape parameter, $\rho$. This graphical test is known as a complementary log-log plot, and a distinct kink in this plot can also indicate a mixture of Weibull distributions. Predictive models for survival times are achieved by relating one or both of the Weibull parameters to functions of the predictor variables. For example, the survReg() function in S-Plus (Insightful Corporation, 2001) models the scale parameter as a log-linear model $\log \kappa_{i}=\mathbf{x}_{(s) i}^{\prime} \beta$, where $\mathbf{x}_{(s) i}$ is the vector of explanatory variables for the survival analysis, $\boldsymbol{\beta}$ is the vector of regression coefficients and a common shape parameter is fitted as well.

\section{Fitting a mixture of parametric survival models}

The two phases (late versus early mortality) can be modeled as being an unobserved binary indicator variable,

$$
q_{i}= \begin{cases}0 & \text { early phase } \\ 1 & \text { late phase, }\end{cases}
$$

with consequent density and survivor functions $f\left(t_{i} \mid q_{i}=1\right)$ or $f\left(t_{i} \mid q_{i}=0\right)$ and $S\left(t_{i} \mid q_{i}=1\right)$ or $S\left(t_{i} \mid q_{i}=0\right)$. The scale parameter is modeled as $\log \kappa_{i}=\mathbf{x}_{(s) i}^{\prime} \beta+\delta q_{i}$, where $\delta$ is the parameter to measure the effect of the phase (late versus early). The shape parameter $\rho$ is assumed constant across all covariate values and phases.

For a mixture distribution of two components, we write the density function as

$$
f\left(t_{i}\right)=\pi_{i} f\left(t_{i} \mid q_{i}=1\right)+\left(1-\pi_{i}\right) f\left(t_{i} \mid q_{i}=0\right)
$$

and the survivor function as

$$
S\left(t_{i}\right)=\pi_{i} S\left(t_{i} \mid q_{i}=1\right)+\left(1-\pi_{i}\right) S\left(t_{i} \mid q_{i}=0\right)
$$

The observed likelihood is evaluated as

$$
L_{O}=\prod_{i=1}^{n} L_{i}
$$

where

$$
L_{i}= \begin{cases}f\left(t_{i}\right) & \text { death at time } t_{i} \\ S\left(t_{i}\right) & \text { censored at time } t_{i}\end{cases}
$$

and hence the (observed) likelihood is

$$
\begin{aligned}
L_{O}= & \prod_{i \in D}\left[\pi_{i} f\left(t_{i} \mid q_{i}=1\right)+\left(1-\pi_{i}\right) f\left(t_{i} \mid q_{i}=0\right)\right] \\
& \times \prod_{i \in C}\left[\pi_{i} S\left(t_{i} ; \mid q_{i}=1\right)+\left(1-\pi_{i}\right) S\left(t_{i} \mid q_{i}=0\right)\right]
\end{aligned}
$$

where $D$ is the set of dead animals and $C$ is the set of censored animals, that is, the animals that had not died by the time the experiment was terminated.

In addition to fitting a survival analysis model for the time to death conditional on the mortality phase $\left(t_{i} \mid q_{i}\right)$, another model for the mortality phase itself $\left(q_{i}\right)$ is required. This is performed by fitting a logistic regression model,

$$
\log \left(\frac{\pi_{i}}{1-\pi_{i}}\right)=\mathbf{x}_{(p) i}^{\prime} \gamma
$$

where $\pi_{i}=\operatorname{Pr}\left\{q_{i}=1\right\}$, the probability of a late mortality phase, $\mathbf{x}_{(p) i}$ is the vector of explanatory variables for the mortality phase, some of which may be in common with $\mathbf{x}_{(s) I}$ and $\gamma$ is the vector of regression coefficients.

\section{Analysis of QTL effects}

For the survival analysis submodel, a full model including mortality phase, the effect of the chromosomal region on MMU1 (QTL1), the effect of the chromosomal region on MMU5 (QTL5), the effect of the chromosomal region on MMU17 (QTL17), a two-way interaction between mortality phase and QTL, the sex (two levels), the background (C57BL/ 6 donor) genotype, the age at challenge and the batch (three levels) was fitted using records of individual animals. Similarly, the submodel for mortality phase (logistic regression) included terms for QTL1, QTL5, QTL17, background genotype, sex, age at challenge and batch. QTL effects were tested as two Wald $z$-tests, b/se(b), using the information of the regression parameter estimates from the survival analysis submodel. Since the genotype QQ was the reference level, then the regression coefficients for $\mathrm{QTL}_{\mathrm{Oq}}$ and $\mathrm{QTL}_{\mathrm{qq}}$ assess the comparisons of the $\mathrm{Qq}$ versus $\mathrm{QQ}$ and qq versus $Q Q$ genotypes.

Fitting reduced models without the variable of interest, and performing a likelihood ratio test were used to assess statistical significance of each of these variables. While testing for a variable in the mortality phase submodel, the term was dropped from this submodel, but retained in the survival time submodel. The revised parameter estimates from the mortality phase submodel were used to calculate the revised $\pi_{i}$ and the revised estimates from the survival submodel used to calculate the revised $f\left(t_{i}\right)$ or $S\left(t_{i}\right)$. The latter was then used to calculate the revised reduced model likelihood $\left(L_{0}\right)$, which was then compared with the 'full model' likelihood $\left(L_{1}\right)$ calculated without the removal of the specific term in the submodel. The likelihood ratio test is calculated as $2 \log \left(L_{1} / L_{0}\right)$. Here, QTL1, QTL5 and QTL17 are three-level factor variables indexing the QTL genotype at each of the chromosomal locations, and 'background genotype' is analyzed using a logit-transformed scale, $\log \left[\left(p_{\mathrm{BG}}+0.01\right) /\left(1-p_{\mathrm{BG}}+0.01\right)\right]$, where $p_{\mathrm{BG}}$ 
is the C57BL/ 6 donor background genotype expressed as a proportion. Logit transformations are commonly used for working with proportions, and in this instance was used to separate out the eight strains with relatively low values of $p_{\mathrm{BG}}$ (see Table 1), allowing any differential effect to be assessed.

The likelihood expression for this mixture model is maximized using the Expectation-Maximization (E-M) algorithm (McLachlan and Basford, 1988; Jansen, 1993). This maximization is performed iteratively, by fitting a survival submodel and a mortality phase (logistic regression) submodel at each iteration.

The additive effect of each QTL was calculated by halving the difference between the homozygotes for C57BL/ 6 donor allele and those for A/J recipient allele. The dominance effect was assessed by contrasting heterozygotes with half the sum of the estimates of the two homozygotes.

During the exploratory stages of the analysis, a term for heterozygosity, $\log [(H+0.01) /(1-H+0.01)]$, was also added to both submodels, to allow for the possible effect of heterosis. However, (logit-transformed) genetic background and heterozygosity are highly correlated, and consequently heterozygosity was dropped from the model, given that background was included. In addition, since background was statistically a far more significant predictor of survival, heterozygosity was subsequently dropped from the models.

\section{Results}

\section{Exploratory analyses}

Figure 1 shows a complementary log-log plot of the survivor function, $\log [-\log \hat{S}(t)]$ versus $\log (t)$, for each group. From this figure, two phases of mortality are clearly apparent from the kinks in the plots: early (usually under 20 days) versus late (over 20 days). It is

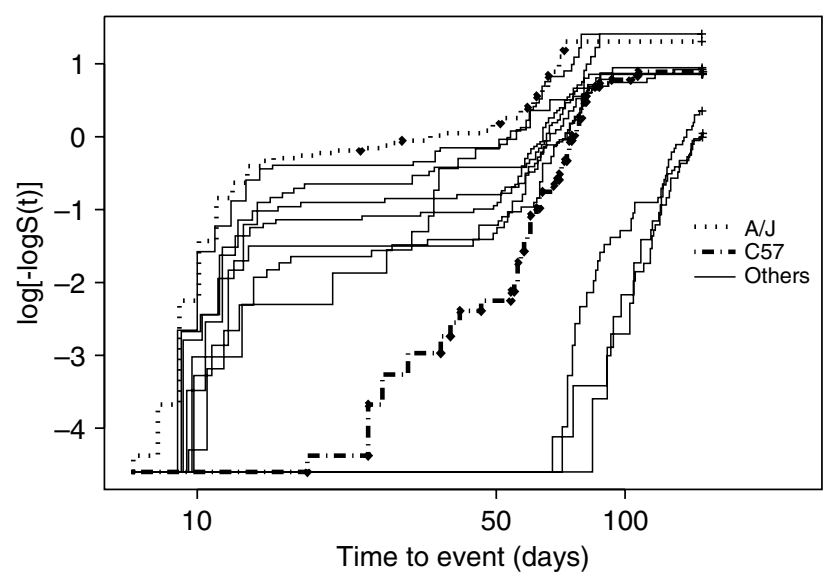

Figure 1 Complementary log-log plots of the empirical survivor functions $S(t)$ versus time since challenge shown on a logarithmic scale. These are shown separately for each genotype group (only donor and recipient strains identified). The mortality phases are indicated by the steep early and late rises, although the timing and size vary between genotype groups. It is worth noting that three of the genotype groups (3,6 and 9) do not fall in the envelope between the sensitive A/J and tolerant C57/BL strains, indicating additional trypanotolerance of these groups. A small separation between groups ( $<1$ day) has been added to facilitate visual separation. apparent that groups 3, 6, 9 and C57BL/6 were almost entirely late phase. Within each phase, the plot is relatively linear, indicating that the Weibull model is appropriate. Further, the curves are relatively parallel within each phase, suggesting that fitting a model with a common shape parameter across all groups is appropriate. Consequently, differences between groups can adequately be explained by differences in the Weibull scale parameters and the proportion of animals belonging to each phase.

To assess the fit of the Weibull mixture model, the empirical survivor functions can be compared with the model-based survivor functions, using the Weibull and logistic parameters as estimated from the data. For this assessment, only group and mortality phase were included in the model; age at challenge, sex and batch were not included. Note that the fitted mixture model is always located between the two component Weibull distributions, approaching asymmetrically the components for small (early phase) and large (late phase) times. Overall, the fits are reasonable, in terms of capturing the main features of the distributions, if not in exact detail. They are considered satisfactory for the current exploratory purposes.

\section{Mortality phase submodel}

Fitting the logistic regression for prediction of mortality phase $(0=$ early death; $1=$ late death) showed that there is little evidence for an effect of the three identified chromosomal regions (Table 2). Only the region on MMU1 showed some association $(P=0.053)$, with the homozygous A/J genotype (qq) having an odds ratio of $0.38 \times$ of being late phase, compared with the homozygous C57BL/6 (QQ). The percentage of donor C57BL/6 genotype, however, was significantly-and positivelyassociated with the odds of being late phase, suggesting that there are additional unidentified genes affecting mortality phase. In addition, males were significantly less likely than females $(P=0.000)$ to be late phase (odds ratio $0.38 \times$ ), and the odds of being late phase reduced significantly with increasing age

Table 2 Likelihood ratio test (LRT) for factors included in the mortality phase submodel while maintaining the full survival time submodel $^{\mathrm{a}}$

\begin{tabular}{lrcc}
\hline Term & LRT & d.f. & $P$ \\
\hline QTL1 & 5.86 & 2 & 0.053 \\
QTL5 & 1.46 & 2 & 0.482 \\
QTL17 & 3.77 & 2 & 0.152 \\
Background & 20.95 & 1 & 0.000 \\
Sex & 19.94 & 1 & 0.000 \\
Age & 8.27 & 1 & 0.004 \\
Batch & 5.52 & 2 & 0.063
\end{tabular}

Terms QTL1, QTL5 and QTL17 refer to the three chromosomal regions and each of these LRTs examines the equality of phenotype effects of the QQ, Qq and qq genotypes. Background refers to the effect of the (logit-transformed) proportion of the donor genotype, and batch refers to the effect of the three separate challenge periods. For further explanation of the full survival time submodel, see the caption in Table 3.

${ }^{a}$ Full survival time submodel: survival $=$ phase+QTL1+QTL5+ QTL17+phase $\times$ QTL1+phase $\times$ QTL5+phase $\times$ QTL17+age+sex+ background+batch. 
$(P=0.004)$. Marginal differences $(P=0.063)$ were observed between the three challenge batches.

\section{The survival analysis submodel}

Fitting the survival model using the Weibull distribution, highly significant interactions $(P=0.000)$ between mortality phase and QTL were detected, indicating that there are separate mortality phase effects for each QTL (Table 3). The effect of an animal dying in the late phase is to increase the mean survival time in the range $3.3 \times$ to $15.8 \times$ depending on the QTL genotype, the most extreme case being for the heterozygous QTL on MMU17 (Table 4).

While there is no effect in the early mortality phase of the QTL on MMU1 and MMU5, homozygous mice for

Table 3 Likelihood ratio test (LRT) for factors included in survival time submodel, while maintaining the full mortality phase submodel $^{\mathrm{a}}$

\begin{tabular}{lrcc}
\hline Term & LRT & d.f. & $\mathrm{P}$ \\
\hline Phase $\times$ QTL1+phase $\times$ QTL5+phase $\times$ QTL17 & 46.35 & 6 & 0.000 \\
QTL1+phase $\times$ QTL1 & 64.83 & 4 & 0.000 \\
QTL5+phase $\times$ QTL5 & 59.51 & 4 & 0.000 \\
QTL17+phase × QTL17 & 101.45 & 4 & 0.000 \\
Background & 31.32 & 1 & 0.000 \\
Sex & 1.31 & 1 & 0.252 \\
Age & 19.78 & 1 & 0.000 \\
Batch & 33.63 & 2 & 0.000
\end{tabular}

The term labeled 'phase $\times$ QTL1+phase $\times$ QTL5+phase $\times$ QTL17' consists of three interactions, and this is used to assess if the effect of the three QTL regions is the same in the early and late phases. Given the significance of these three interaction terms $(P=0.000)$, the following three LRTs ('QTL1+phase $\times$ QTL1', 'QTL5+phase $\times$ QTL5' and 'QTL17+phase $\times$ QTL17') are used to assess the significance of each QTL region, allowing for different effects in early and late phases. For further explanation of the terms and the full mortality phase submodel, see the caption in Table 2.

${ }^{a}$ Full mortality phase submodel: phase=QTL1+QTL5+QTL17+ age+sex+background+batch.

Table 4 Predicted QTL effect: survival mean and standard deviation (days post-inoculation)

\begin{tabular}{|c|c|c|c|c|c|c|c|}
\hline \multirow{2}{*}{$\begin{array}{l}\text { Mortality } \\
\text { phase }\end{array}$} & \multirow{2}{*}{$\begin{array}{c}\text { QTL } \\
\text { genotype }\end{array}$} & \multicolumn{2}{|c|}{ MMU1 } & \multicolumn{2}{|c|}{ MMU5 } & \multicolumn{2}{|c|}{ MMU17 } \\
\hline & & Mean & s.d. & Mean & s.d. & Mean & s.d. \\
\hline \multirow[t]{3}{*}{ Early } & QQ & 15.48 & 5.53 & 16.07 & 5.74 & 18.97 & 6.77 \\
\hline & $\widehat{\mathrm{Qq}}$ & 15.00 & 5.36 & 15.81 & 5.65 & 5.78 & 2.07 \\
\hline & $\mathrm{qq}$ & 14.35 & 5.12 & 14.94 & 5.34 & 5.33 & 1.90 \\
\hline \multirow[t]{3}{*}{ Late } & QQ & 74.10 & 26.46 & 76.94 & 27.48 & 90.80 & 32.43 \\
\hline & $\hat{\mathrm{Qq}}$ & 76.31 & 27.25 & 69.88 & 24.96 & 91.29 & 32.60 \\
\hline & $\mathrm{qq}$ & 51.90 & 18.53 & 49.71 & 17.75 & 55.28 & 19.74 \\
\hline \multirow[t]{3}{*}{ Overall } & QQ & 71.28 & 28.73 & 73.05 & 30.50 & 76.00 & 41.09 \\
\hline & $\mathrm{Qq}$ & 72.35 & 30.39 & 67.29 & 26.98 & 86.43 & 37.34 \\
\hline & $\mathrm{qq}$ & 47.45 & 21.28 & 46.90 & 19.54 & 52.66 & 22.22 \\
\hline
\end{tabular}

$\mathrm{Q}=$ donor allele $(\mathrm{C} 57 \mathrm{BL} / 6), \mathrm{q}=$ recipient allele $(\mathrm{A} / \mathrm{J})$. Results for early and late mortality phases were obtained by applying the estimated regression coefficients of the survival time submodels for each phase $\times$ QTL combination while specifying mean values for all other terms in the model, and using results for the mean and standard deviation of Weibull distributions.

The overall results refer to the values for the mixture distribution (early and late phases), where the mixture probabilities were obtained from the estimated regression coefficients of the mortality phase model. See Appendix A for details of methods. the C57BL/6 donor QTL allele on MMU17 show longer survival time $(P<0.01)$ than mice homozygous for the recipient $A / J$ allele and heterozygous mice. The recipient A/J QTL allele shows complete dominance for survival at this phase with dominance effect $(d)$ equal to -6.6 days and the additive effect being 6.82 days.

In contrast, there are substantial effects of all the three chromosomal regions on the late mortality phase. On the basis of the parameter estimates (results not shown) and the means in Table 4 , the donor C57BL/6 alleles show dominance (with increased survival time) for MMU1 ( $d=13.3$ days) and MMU17 ( $d=18.25$ days), with no apparent dominance for MMU5.

For a given mortality phase, no difference in mean survival time of males and females was detected $(P=0.252)$, whereas increasing age results in a significant increase in mean survival time $(P=0.000)$. Considering both mortality phase and survival time submodels with and without age confirmed this result. Mainly, not including age in the survival time submodel left the estimated effect of age unchanged in the mortality phase submodel, and vice versa. In addition, increased amounts of donor C57BL/6 genotype were associated with significant increase in mean survival time $(P=0.000)$. Batch differences in survival times were also observed $(P=0.000)$. As a further analysis, the estimated phase effect with and without sex in the survival model had no real effect on the regression parameter estimate of the phase effect (results not shown).

\section{The combined model}

By combining the results from the mortality phase and survival analysis submodels, the overall means (and standard deviations) for each QTL genotype averaged over both phases can be calculated, as shown in Table 4 . In addition, the fitted probability density functions for survival time can also be calculated, and these are shown in Figure 2. It is worth noting that in the calculations in both Table 4 and Figure 2, the distributions have been adjusted to the mean levels of the other covariates, in both survival and mortality phase submodels. Consistent with the results of the submodels, the donor C57BL/6 alleles-either in heterozygous or homozygous formconvey increased survival to the animals.

From Figure 2, it appears that the three QTL had markedly different effects on the proportion of mice in the early phase versus late phase of survival. For QTL1 and QTL5, the heterozygous $\mathrm{Qq}$ and homozygous C57BL/6 (QQ) genotypes had a higher proportion of animals in the late phase of survival. In contrast, for QTL17, the C57BL/6 (QQ) genotype had markedly lower proportion of animals in the late phase of survival than the heterozygous $(\mathrm{Qq})$ and the $\mathrm{A} / \mathrm{J}(\mathrm{qq})$ genotypes.

It should be noted that the models developed here are for unrelated individuals, and that is not the case in the current data. As a consequence, the reported $P$-values are more significant than they should be. However, where results are significant, they are usually very highly significant (see Tables 2 and 3), so an analysis taking into account the pedigree structure would essentially give the same results.

Overall, the fits of models are satisfactory as can be seen in Table 5 where the distribution of the observed and estimated number of mice over different mortality 


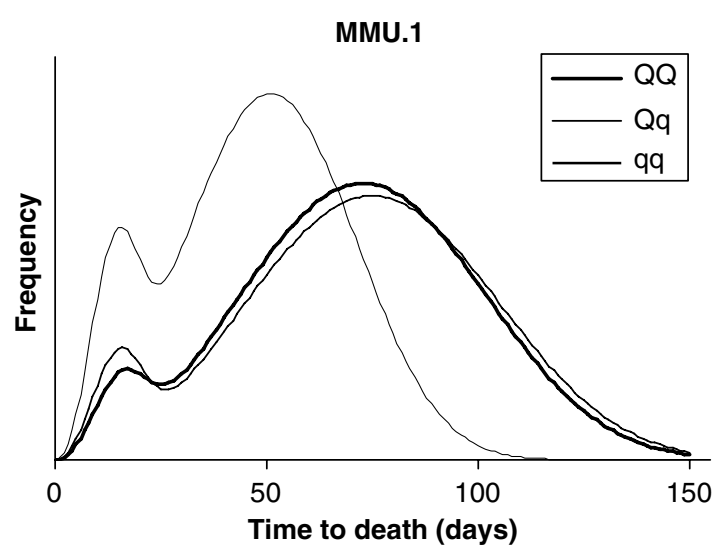

\begin{tabular}{cccc}
\hline $\begin{array}{c}\text { Para- } \\
\text { meters }\end{array}$ & QQ & Qq & \multicolumn{1}{c}{ qq } \\
\hline$\kappa_{1}$ & 82.90 & 85.37 & 58.06 \\
$\kappa_{2}$ & 17.32 & 16.78 & 16.05 \\
$\pi$ & 0.95 & 0.93 & 0.88 \\
\hline
\end{tabular}

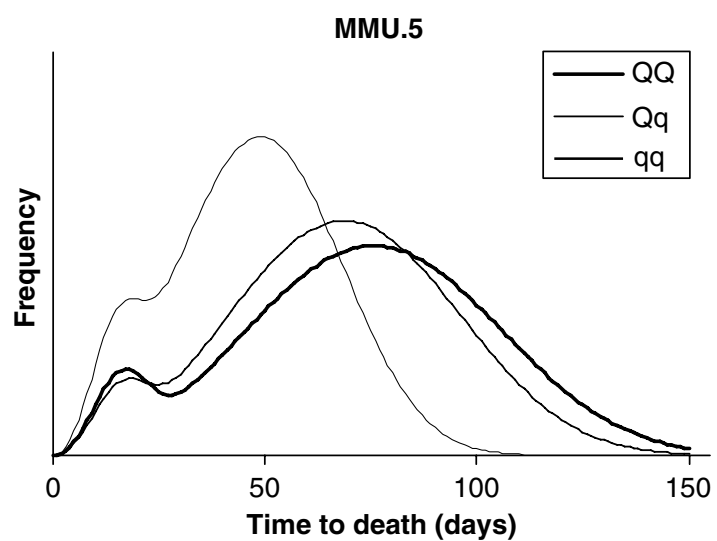

\begin{tabular}{crrr}
\hline $\begin{array}{c}\text { Para- } \\
\text { meters }\end{array}$ & QQ & Qq & \multicolumn{1}{c}{ qq } \\
\hline$\kappa_{1}$ & 86.08 & 78.19 & 55.62 \\
$\kappa_{2}$ & 17.98 & 17.69 & 16.72 \\
$\pi$ & 0.94 & 0.95 & 0.92 \\
\hline
\end{tabular}

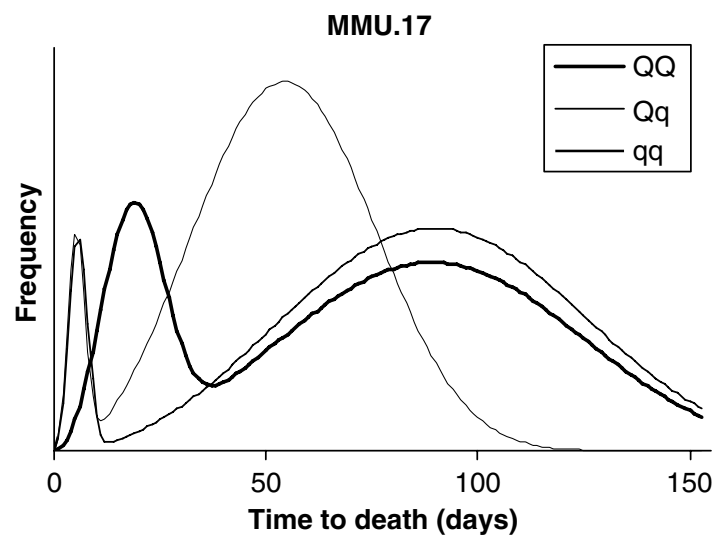

\begin{tabular}{crrr}
\hline $\begin{array}{c}\text { Para- } \\
\text { meters }\end{array}$ & QQ & Qq & \multicolumn{1}{c}{ qq } \\
\hline$\kappa_{1}$ & 101.60 & 102.14 & 61.85 \\
$\kappa_{2}$ & 21.22 & 6.47 & 5.97 \\
$\pi$ & 0.79 & 0.94 & 0.95 \\
\hline
\end{tabular}

Figure 2 Predicted frequency distribution of survival over time: QTL effect at each chromosome $(\rho=3.06=$ constant shape parameter, $\kappa_{1}=$ scale parameter in late mortality phase, $\kappa_{2}=$ scale parameter in early mortality phase, $\pi=$ proportion of mice in the late mortality phase). $\mathrm{Q}$, donor allele $(\mathrm{C} 57 \mathrm{BL} / 6) ; \mathrm{q}$, recipient allele $(\mathrm{A} / \mathrm{J})$.

phases and different QTL genotype groups are displayed.

\section{Discussion}

Figure 1 clearly shows two mortality phases in the current experiment, early versus late death. To our knowledge, this is the first report describing such a biphasic survival pattern. The occurrence of early death (within 20 days post-inoculation) could be the result of a failure of the host defense system to cope with the early infection. Animals that survive this first assault of parasite infection might have developed resistance by means of antibodies that maintain a balance between the parasites and the host. The ability to cope with the first infection assault could be related to a genetic character of survivors. However, trypanosomes have the ability to change their surface antigen (variable surface glycoprotein) during infection to escape the immune response (Vickerman and Luckins, 1969; Cross, 1975; Inverso et al., 1988). By the principle of homeostasis, the host develops antibodies against the new antigens to re-establish equilibrium. Although there is no evidence of a biological explanation for the observed late death, the continuous disruption of this unstable equilibrium due to changes in parasite surface antigen (Vickerman and Luckins, 1969; Cross, 1975; Inverso et al., 1988) and the effort of the host to maintain this equilibrium that might 
Table 5 Distribution of the observed and expected number of individuals through QTL genotype groups and mortality phase

\begin{tabular}{|c|c|c|c|c|c|c|c|}
\hline \multirow[t]{2}{*}{ Mortality phase } & \multirow[t]{2}{*}{ QTL genotype } & \multicolumn{2}{|c|}{ MMU1 } & \multicolumn{2}{|c|}{ MMU5 } & \multicolumn{2}{|c|}{ MMU17 } \\
\hline & & $\operatorname{Obs}(20)^{\mathrm{a}}$ & $\operatorname{Exp}(\pi)^{\mathrm{b}}$ & $O b s(20)$ & $\operatorname{Exp}(\pi)$ & Obs (20) & $\operatorname{Exp}(\pi)$ \\
\hline \multirow[t]{4}{*}{ Early } & QQ & 13 & 11.6 & 23 & 21.7 & 3 & 15.5 \\
\hline & $\widehat{\mathrm{Qq}}$ & 23 & 22.9 & 13 & 12.4 & 22 & 21.9 \\
\hline & $\mathrm{qq}$ & 132 & 144.9 & 132 & 145.3 & 143 & 141.9 \\
\hline & Total & 168 & 179.3 & 168 & 179.3 & 168 & 179.3 \\
\hline \multirow[t]{4}{*}{ Late } & QQ & 181 & 182.4 & 185 & 186.3 & 135 & 122.5 \\
\hline & $\mathrm{Qq}$ & 160 & 160.1 & 152 & 152.6 & 148 & 148.1 \\
\hline & qq & 222 & 209.1 & 226 & 212.7 & 280 & 281.1 \\
\hline & Total & 563 & 551.7 & 563 & 551.7 & 563 & 551.7 \\
\hline \multirow[t]{4}{*}{ Overall } & QQ & 194 & 194 & 208 & 208 & 138 & 138 \\
\hline & $\mathrm{Qq}$ & 183 & 183 & 165 & 165 & 170 & 170 \\
\hline & $\mathrm{qq}$ & 354 & 354 & 358 & 358 & 423 & 423 \\
\hline & Total & 731 & 731 & 731 & 731 & 731 & 731 \\
\hline
\end{tabular}

${ }^{\mathrm{a}} \mathrm{Obs}(20)$ refers to the number of mice that died before 20 days (early) versus 20 days or more (late).

${ }^{\mathrm{b}} \operatorname{Exp}(\pi)$ refers to the expected number of mice that died in the early versus late phase, as estimated from the model-based probabilities $\left(\hat{\pi}_{i}\right)$. They are determined by summing the estimated probabilities for all observations in a particular QTL genotype group: $\operatorname{Exp}(\pi)=\sum_{i \in \mathrm{QTLGenotype}} \hat{\pi}_{i}$.

be energy consuming could explain the observed late death.

Mortality phase has shown to be an important factor in the survival analysis. Indeed, the inclusion of this variable into the survival model has enabled the effect of trypanotolerance QTL to be assessed separately in early and late mortality phases. Interestingly, it has allowed us to discover that trypanotolerance QTL on MMU17 acts on both mortality phases in an opposite way: recessive in the early phase, the donor C57BL/6 QTL allele shows a complete dominance in the late mortality phase (Figure 2). This is one of the major findings resulting from the current analysis and based on the method that has been described in this paper.

The QTL for trypanotolerance in mice was first localized by Kemp et al. (1997). They used two types of F2 populations to estimate the expected differences in survival time between mice homozygous for the resistant allele and those homozygous for the susceptible allele. The cross between the susceptible BALB/C strain and C57BL/ 6 resulted in a difference of 32, 22 and 36 days for MMU1, MMU5 and MMU17 respectively. The F2 population originating from the susceptible $\mathrm{A} / \mathrm{J}$ strain and C57BL/ 6 resulted in difference of 22 and 31 days for MMU5 and MMU17, whereas no significant effects due to MMU1 were found. Koudandé et al. (2005) performed a survival analysis using the product-limit method also known as the Kaplan-Meier method and estimated that mice homozygous for the C57BL/6 allele on the QTL on MMU1, MMU5 and MMU17 on average lived 20.2, 19.8 and 17.1 days longer than the $\mathrm{A} / \mathrm{J}$ mice. These results are in line with those in the original mapping population but the estimated effects are on average $30 \%$ smaller. They explained the smaller effects by the difference in the background on which the effects of QTL effects were tested. In our analysis, the combined model revealed that mice homozygous for the C57BL/6 allele on the QTL on MMU1, MMU5 and MMU17 on average lived 23.8, 26.2 and 23.3 days longer than the $\mathrm{A} / \mathrm{J}$ mice. These estimates are larger than those found by Koudandé et al. (2005). In fact, in the current analysis, observations on the animals from the heterozygous groups were also utilized in estimating the effects in the model. This is a further result of the current survival analysis model.
Kemp et al. (1997) found that the donor C57BL/6 QTL on MMU17 had the largest effect on survival and explained $14.6 \%$ of the total variation in an $\mathrm{F} 2$ cross of $\mathrm{C} 57 \mathrm{BL} / 6 \times \mathrm{BALB} / \mathrm{c}$ and $9 \%$ in an $\mathrm{F} 2$ cross $\mathrm{C} 57 \mathrm{BL} / 6 \times \mathrm{A} / \mathrm{J}$. In our survival analysis, the additive effects were 17.6, 21.5 and $24.3 \%$ of the mean value of homozygous mice respectively for QTL1, QTL5 and QTL17 in the late mortality phase. The survival analysis method developed for this study is more appropriate, and also extracts more information from the data than that used in Kemp et al. (1997). In particular, the survival analysis method correctly addresses the issue of censored data, rather than assuming that the survival time is the last day of the experiment +1 . In addition, it addresses the issue of the biphasic mortality pattern, which was not an issue in the other studies. The survival analysis method, however, does not provide results in terms of percentage of variance explained, as it is not a linear model under the assumption of normally distributed errors.

Trypanotolerance QTL on MMU1 and MMU17 appear to have dominant effects in late mortality phase on survival while the effect tended toward dominance or additive on MMU5. This result is in contrast to earlier studies in which the donor C57BL/6 QTL on MMU17 and MMU5 tended toward partially recessive effects on average survival (Kemp et al., 1997). However, our result on MMU17 in the early phase is in agreement with that of these authors.

The observations on the effect of sex are particularly interesting in that in all previous work on trypanosome challenge of A/J, C57BL/ 6 and their crosses, substantial effects of sex on average survival have been observed (Kemp SJ, personal communication). These are now revealed to be an effect on proportion of mice in the two mortality phases, rather than an effect on average survival within a mortality phase. This result demonstrates that applying our extended model provides a better understanding of the effect of QTL.

This is the first study on the effects of chromosomal regions moved into a new genetic background on survival patterns. From this study, we found clear evidence for a biphasic survival pattern and QTL have different effects on different mortality phases. We provide models to analyze such survival patterns. These models can also be used when studying defense 
mechanisms against other pathogens. Finally, these approaches provide further information on the nature of gene actions.

\section{Acknowledgements}

We are grateful to The Netherlands Foundation for the Advancement of Tropical Research (WOTRO) and ILRI for financial support as well as providing laboratory facilities. We acknowledge the two anonymous reviewers whose comments have helped to improve the manuscript. ODK acknowledges the leave provided by INRAB (Institut National des Recherches Agricoles du Bénin) to carry out this research.

\section{References}

Clapcott SJ, Teale AJ, Kemp SJ (2000). Evidence for genomic imprinting of the major QTL controlling susceptibility to trypanosomiasis in mice. Parasite Immunol 22: 259-263.

Cross GA (1975). Identification, purification and clone-specific glycoprotein antigens constituting the surface coat of Trypanosoma brucei. Parasitology 71: 393-417.

Insightful Corporation (2001). S-Plus 6 Guide to Statistics. vol. 1-2. Insightful Corporation: Seattle, WA.

Inverso JA, de Gee ALW, Mansfield JM (1988). Genetics of resistance to the African trypanosomes: VII. Trypanosome virulence is not linked to variable surface glycoprotein expression. J Immunol 140: 289-293.

Iraqi F, Clapcott SJ, Kumari P, Haley CS, Kemp SJ, Teale AJ (2000). Fine mapping of trypanosomiasis resistance loci in murine advanced intercross lines. Mamm Genome 11: 645-648.

Jansen RC (1993). Maximum likelihood in a generalized linear finite mixture model by using the EM algorithm. Biometrics 49: 227-231.

Jennings FW, Whitelaw DD, Holmes PH, Urquhart GM (1978). The susceptibility of strains of mice to infection with Trypanosoma congolense. Res Vet Sci 25: 399-400.

Kalbfleisch JD, Prentice RL (1980). The Statistical Analysis of Failure Time Data. John Wiley \& Sons: New York.

Kemp SJ, Darvasi A, Soller M, Teale AJ (1996). Genetic control of resistance to trypanosomiasis. Vet Immunol Immunopathol 54: 239-243.

Kemp SJ, Iraqi F, Darvasi A, Soller M, Teale AJ (1997). Localization of genes controlling resistance to trypanosomiasis in mice. Nat Genet 16: 194-196.

Koudandé OD, van Arendonk JAM, Iraqi F (2005). Marker assisted introgression of trypanotolerance QTL in mice. Mamm Genome 16: 112-119.

Masake RA, Musoke AJ, Nantulya VM (1983). Specific antibody responses to the variable surface glycoproteins of Trypanosoma congolense in infected cattle. Parasite Immunol 5: 345-355.

McLachlan GJ, Basford KE (1988). Mixture Models: Inference and Applications to Clustering. Dekker: New York.
Morrison WI, Murray M (1979). Trypansoma congolense: inheritance of susceptibility to infection in inbred strains of mice. Exp Parasitol 48: 364-374.

Morrison WI, Roelants GE, Mayor-Withey KS, Murray M (1978). Susceptibility of inbred strains of mice to Trypanosoma congolense: correlation with changes in spleen lymphocyte populations. Clin Exp Immunol 32: 25-40.

Roberts CJ, Gray AJ (1973). Studies on trypanosome-resistant cattle II. The effects of trypanosomiasis on N'Dama, Muturu and Zebu cattle. Trop Anim Health Prod 5: 220-233.

Roelants GE (1986). Natural resistance to African trypanosomiasis. Parasite Immunol 8: 1-10.

Stam P, Zeven AC (1981). The theoretical proportion of the donor genome in near-isogenic lines of self-fertilizers bred by backcrossing. Euphytica 30: 227-238.

Teale A, Agaba M, Clapcott S, Gelhaus A, Haley C, Hanotte $\mathrm{O}$ et al. (1999). Resistance to trypanosomiasis: of markers, genes and mechanisms. Arch Tierz Dummerstorf 42: 36-41.

Vickerman K, Luckins AG (1969). Localization of variable antigens in the surface coat of Trypanosoma brucei using ferritin conjugated antibody. Nature 224: 1125-1126.

\section{Appendix A}

For each QTL, the mean and standard deviation of survival time for those in the early or late mortality phase are presented (Table 4). This has been determined by calculating the estimated Weibull scale parameter, $\hat{\kappa}_{j k l}=\exp \left(\mathbf{x}_{(s) j k l}^{\prime} \hat{\boldsymbol{\beta}}_{(s)}\right)$ by setting the elements of $\mathbf{x}_{(s) j k l}$ to indicate early or late phase (j), QTL genotype QQ, Qq or qq $(k)$ for the particular QTL region $(l)$ (on either MMU1, MMU5 or MMU17), with all other elements of $\mathbf{x}_{(s) j k l}$ set to their mean values for their respective predictor variables. Using the estimated common shape parameter, $\hat{\rho}$, the mean and standard deviations were calculated as $\hat{\mu}_{j k l}=\hat{\kappa}_{j k l} \Gamma(1+1 / \hat{\rho})$ and $\hat{\sigma}_{j k l}=\hat{\kappa}_{j k l}\left\{\Gamma(1+2 / \hat{\rho})-[\Gamma(1+1 / \hat{\rho})]^{2}\right\}^{1 / 2}$ using standard results for a Weibull distribution, where $\Gamma(\cdot)$ is the gamma function. The overall means and standard deviations have been calculated by combining over early and late phases. This first requires estimation of the probabilities of late phase mortality from the logistic regression model, $\hat{\pi}_{k l}=\left[1+\exp \left(-\mathbf{x}_{(p) k l}^{\prime} \hat{\boldsymbol{\beta}}_{(p)}\right)\right]^{-1}$, where $\mathbf{x}_{(p) k l}$ is set up in a similar manner to $\mathbf{x}_{(s) j k l}$, thus giving a set of late phase probabilities for each genotype/QTL combination. Next, the overall means and standard deviations are estimated using standard mixture model results, namely $\hat{\mu}_{\cdot k l}=\hat{\pi}_{k l} \hat{\mu}_{1 k l}+\left(1-\hat{\pi}_{k l}\right) \hat{\mu}_{2 k l}$ and $\sigma_{\cdot k l}=$ $\left\{\hat{\pi}_{k l}\left(\hat{\mu}_{1 k l}-\hat{\mu}_{\cdot k l}\right)^{2}+\left(1-\hat{\pi}_{k l}\right)\left(\hat{\mu}_{2 k l}-\hat{\mu}_{\cdot k l}\right)^{2}+\hat{\pi}_{k l} \hat{\sigma}_{1 k l}^{2}+\left(1-\hat{\pi}_{k l} \hat{\sigma}_{2 k l}^{2}\right\}^{1 / 2}\right.$. 